\section{Carcinoma tímico en niños. Informe de dos casos}

\section{RESUMEN}

El carcinoma tímico es una neoplasia poco frecuente en la población pediátrica. El diagnóstico diferencial se realiza con tumores mediastinales. Generalmente las manifestaciones clínicas se deben a obstrucción de la vía aérea. El tratamiento incluye cirugía, quimioterapia y radioterapia. Los esquemas de tratamiento, especialmente la quimioterapia, es motivo de controversia en niños y la mayoría de los protocolos se basan en la experiencia obtenida de los adultos. El pronóstico es malo, con pocas posibilidades de curación, depende del estadio según la clasificación de Masaoka y de la posibilidad de resección quirúrgica.

En este informe se describen dos casos de carcinoma tímico en niños: uno de 12 años cuya afección se inició con cianosis e hipercalcemia y en una paciente de 14 años en quien empezó con tos y dolor torácico.

Palabras clave: carcinoma tímico, quimioterapia, clasificación, pronóstico, oncología pediátrica.

\section{Thymic carcinoma in pediatric population: report of two cases}

\section{ABSTRACT}

Thymic carcinoma is a rare tumor in pediatric population. Differential diagnoses include mediastinal tumors. Most clinical manifestations are due to obstruction. Treatment includes surgery, chemotherapy and radiotherapy. The treatment schemes, especially chemotherapy, are controversial in children, and most of them are based on the experience with adult patients. In general, thymic carcinoma has a poor prognosis, with low chances for cure, and it is based on Masaoka et al classification and the possibility of surgical resection.

We report two cases of thymic carcinoma in children: a 12-year-old boy who presented with cyanosis and hypercalcemia, and a 14-year-old girl whose inicial symptoms were cough and chest pain.

Key words: thymic carcinoma, chemotherapy, classification, prognosis, pediatric oncology.
Yamile K Chaucanez-Bastidas ${ }^{1}$ Daniel Ortiz-Morales² Pedro Pasquel-García Velarde ${ }^{3}$ Armando B Martínez-Ávalos ${ }^{4}$

${ }^{1}$ Residente del tercer año de pediatría.

2 Oncólogo pediatra.

${ }^{3}$ Patólogo. Médico Adscrito al Servicio de Patología.

${ }^{4}$ Oncólogo Pediatra. Médico Adscrito al Servicio de Oncología.

Instituto Nacional de Pediatría, México.
Recibido: 16 de octubre del 2014

Aceptado: 17 de febrero del 2015

Correspondencia: Dr. Armando B. Martínez Ávalos Servicio de Oncología Pediátrica Instituto Nacional de Pediatría Insurgentes Sur 3700-C CP 04530 México, D.F.

Tel: 10840900, ext. 1339

mtzgro@prodigy.net.mx

\section{Este artículo debe citarse como}

Chaucanez-Bastidas YK, Ortiz-Morales D, PasquelGarcía Velarde P, Martínez-Ávalos AB. Carcinoma tímico en niños. Informe de dos casos. Acta Pediatr Mex 2015;36:456-463. 


\section{INTRODUCCIÓN}

El timo es un órgano complejo conformado por elemento epiteliales y linfoides; es el sitio de maduración de los linfocitos T. ${ }^{1}$ Las neoplasias epiteliales del timo incluyen timomas, carcinoides y carcinoma tímico. Los tímicos son los tumores más comunes de los mediastinales en el adulto $(40 \%))^{2-4}$ en niños representan el $1.5 \%$ de los tumores mediastinales. ${ }^{3,5}$ La edad media de presentación es a los 13 años y predominan en los varones en proporción de 9:5..$^{6-8}$ Stachowicz y sus colaboradores describieron pacientes polacos con edades entre 4.5 y 19 años (media de 16 años). ${ }^{9}$ En el grupo italiano de tumores raros (Proyecto TREP) de nueve pacientes estudiados en 10 años la edad media fue de 12.4 años. ${ }^{10}$

En los carcinomas tímicos las células epiteliales pierden sus características fenotípicas y funcionales, muestran anaplasia celular, con núcleos prominentes, cromatina vesicular, abundante actividad mitósica, aumento del índice núcleocitoplasma y necrosis; infiltración extensa a los tejidos circundantes y metástasis extratorácica; ${ }^{11}$ la mayoría expresa CD5.1,12 El carcinoma tímico fue descrito por primera vez como neoplasia del epitelio tímico en 1977 por Shimosato. ${ }^{13}$

Histopatológicamente hay varios subtipos de carcinoma: de células escamosas (que se clasifica en bien diferenciado o queratinizado, moderadamente diferenciado y pobremente diferenciado; antes carcinoma semejante a linfoepitelioma) asociado con infección por virus de Epstein-Barr ${ }^{13}$ y otros subtipos como carcinoma de células claras mucoepidermoide, carcinosarcoma, anaplásico, entre otros..$^{4,13,14}$ El tipo histológico más común es el de células escamosas cuya variedad poco diferenciada es la más común en la población occidental. ${ }^{13}$

Los síntomas iniciales del tumor mediastinal anterior son dolor torácico, ${ }^{10}$ tos crónica, disnea, fiebre, pérdida de peso, dificultad respiratoria y síndrome de vena cava superior ${ }^{3,9}$ y suelen presentarse de tres semanas a seis meses. Hasta $30 \%$ de los casos son asintomáticos. ${ }^{3,10,15}$

La diferencia de la miastenia grave con el timoma es que se presenta entre 15 a 59\% de los casos (8\% en carcinoma tímico $\left.{ }^{16}\right)$. En niños se han descrito casos relacionados con esclerodermia, lupus eritematoso, osteoartropatía hipertrófica, polimiositis, eritrodermia, síndrome de Sjögren y síndrome nefrótico. ${ }^{9,10,17-19}$

Radiológicamente puede haber derrame pleural e invasión a la pleura y al pericardio. ${ }^{3}$ La tomografía axial computada contrastada es el estudio de elección para los tumores tímicos. Se ha utilizado la tomografía por emisión de positrones con fluorodesoxiglucosa para detectar metástasis pleurales. ${ }^{20}$

De todos los pacientes con carcinoma tímico 46\% tiene metástasis linfáticas. La diseminación hematológica a pulmón, hígado, hueso, riñón y cerebro ocurre en $43,46,29,18$ y $11 \%$, respectivamente. ${ }^{7,21}$

La primera clasificación de los tumores tímicos fue propuesta por Bernatz de manera descriptiva, dependiendo del contenido de células linfocíticas. Levine y Rosai presentaron una clasificación clinicopatológica. ${ }^{1}$ En 1999 la Organización Mundial de la Salud propuso una clasificación histopatológica (Cuadro 1) designando con letras la morfología de las células epiteliales neoplásicas y dependiendo de la relación entre células epiteliales y linfocitos, así como la atipia celular. En esta clasificación los carcinomas tímicos corresponden a B3 y C, con supervivencia a 10 años de 35 y $30 \%$, respectivamente. ${ }^{22}$ El tipo C tiene una prevalencia de 10 a $25 \%^{1}$ (Cuadro 1).

Actualmente se utiliza el sistema de estadificación quirúrgico de Masaoka y su grupo 
Cuadro 1. Clasificación patológica de los timomas según la Organización Mundial de la Salud

\begin{tabular}{lcc}
\hline Tipo & Descripción histológica & $\begin{array}{c}\text { Tasa de supervi- } \\
\text { vencia a 10 años }\end{array}$ \\
A & Timoma medular & $100 \%$ \\
AB & Timoma mixto & $100 \%$ \\
B1 & Timoma predominantemente & $80 \%$ \\
B2 & cortical & $80 \%$ \\
B3 & Timoma cortical & $35 \%$ \\
C & Carcinoma tímico bien & \\
diferenciado & $30 \%$ \\
\hline
\end{tabular}

(Cuadro 2). ${ }^{23}$ Hay una relación muy estrecha entre ambos sistemas de clasificación, los pacientes clasificados como tipos B2, B3 y C corresponden a los estadios III y IV de Masaoka. ${ }^{8,13}$ Otras clasificaciones son las de Müller-Hermelink y la de Suster y Morán, pero actualmente no se usan ${ }^{24,25}$ (Cuadro 2).

El tratamiento del carcinoma tímico en niños se basa en la experiencia obtenida en adultos y en tratamientos heterogéneos de series de casos. El tratamiento debe ser multimodal incluyendo cirugía, quimioterapia y radioterapia. La cirugía

Cuadro 2. Clasificación de Masaoka et $a^{23}$ de los tumores tímicos y su correlación con la clasificación de Suster y Morán ${ }^{11}$

\begin{tabular}{llc}
\hline Masaoka & \multicolumn{1}{c}{ Descripción } & $\begin{array}{c}\text { Suster \& } \\
\text { Moran }\end{array}$ \\
I & $\begin{array}{l}\text { Completamente encapsulado } \\
\text { macroscópicamente; microscópi- } \\
\text { camente sin invasión capsular } \\
\text { Invasión macroscópica al tejido } \\
\text { adiposo circundante o pleura } \\
\text { II A }\end{array}$ & I \\
II B & $\begin{array}{l}\text { Invasión microscópica al tejido } \\
\text { adiposo circundante o pleura } \\
\text { mediastinal } \\
\text { III }\end{array}$ & $\begin{array}{l}\text { Invasión macroscópica a los } \\
\text { órganos vecinos: pericardio, } \\
\text { pulmón y grandes vasos } \\
\text { Diseminación pericárdica o } \\
\text { pleural } \\
\text { IV A }\end{array}$ \\
IV B & $\begin{array}{l}\text { Metástasis linfáticas o } \\
\text { hematógenas }\end{array}$
\end{tabular}

es la piedra angular del tratamiento, aunque puede ser difícil por infiltración local del tumor y metástasis. ${ }^{26}$ La resección tumoral completa ofrece las mejores oportunidades de curación. . $^{3,25-29}$ Los pacientes con tumores en estadio I tratados solamente con resección completa tienen supervivencia a 5 años de $100 \%$, con recurrencia de $1 \% .^{26,30-32}$ La resección subtotal es insuficiente; sólo un tercio de los pacientes a quienes se les realizó biopsia inicialmente viven 10 años. ${ }^{33}$ La resección completa es posible en 10 a $60 \%$ de los pacientes; según Stachowicz-Stencel se logró la resección total en 1 de 9 pacientes. ${ }^{9}$ En caso de recurrencia la cirugía total sigue siendo el arma principal para obtener supervivencias a largo plazo. ${ }^{8}$ El tratamiento quirúrgico utilizado es la esternotomía media ${ }^{34}$ aunque también se puede realizar toracotomía anterolateral. $.27,30,35$

La radioterapia ayuda al control local de la enfermedad. ${ }^{30}$ Su uso en pacientes con carcinoma tímico Masaoka II es motivo de controversia ya que los tumores con infiltración transcapsular pueden tratarse solamente con cirugía. La National Comprehensive Cancer Network sugiere el uso de radioterapia tridimensional o de intensidad modulada posquirúrgica, en los pacientes con carcinoma tímico, para disminuir recaídas locales, ${ }^{36}$ aunque en algunas series no se ha visto beneficio. ${ }^{37}$ Los efectos secundarios de la radioterapia en el mediastino incluyen morbilidad cardíaca, neumonitis, fibrosis pulmonar, estenosis esofágica y segundas neoplasias.

Aunque la resección completa para tumores en estadio III ocurre entre 45 y $55 \%$ se recomienda radioterapia adyuvante. Estos pacientes tratados con altas dosis de radiación tienen supervivencia a 5 años de 30 a 50\%; cuando se realiza resección total y radioterapia la supervivencia es de $60 \%$. Si la resección es subtotal, aún con el uso de radioterapia, la supervivencia es de $30 \%$. Onuki y sus colegas ${ }^{38}$ observaron que con dosis bajas de radioterapia prequirúrgica, más 
resección y radioterapia adyuvante en pacientes en estadios III, la supervivencia a 10 años es de $75 \% .{ }^{38,39}$

El uso de radioterapia en carcinoma tímico depende del estadio y de la extensión de la resección quirúrgica. ${ }^{40}$ La radioterapia en pacientes con estadio IVA está bien establecida. Wright y su gripo señalaron que la radioterapia preoperatoria concomitante con quimioterapia, empleando cisplatino y etopósido, obtiene 40 a $99 \%$ de necrosis de los tumores obtenidos por cirugía. ${ }^{41}$ Nonaka y sus colaboradores mostraron que una dosis de 60 Gy mejora el pronóstico de pacientes con carcinoma tímico en estadios III y IV. ${ }^{42}$ La radioterapia disminuye la recurrencia local y prolonga las tasas de supervivencia. ${ }^{42-44}$ La dosis total de radioterapia utilizada oscila entre 40 y 70 Gy. ${ }^{42}$

La National Comprehensive Cancer Network ${ }^{36}$ propone dosis de 60 a 70 Gy para pacientes con tumores irresecables como tratamiento adyuvante, 45 a 50 Gy para tumores con márgenes negativos y 54 Gy cuando hay márgenes positivos microscópicos. Para enfermedad residual microscópica la dosis debe ser de 60 Gy en fracciones convencionales de 1.8 a 2.0 Gy diarios. ${ }^{42,45}$

Los pacientes con estadios I y los estadios II completamente resecados no se benefician con radioterapia para el control local, recurrencia o supervivencia, después de una resección quirúrgica. ${ }^{40}$

La quimioterapia neoadyuvante está indicada cuando la resección completa no es posible y se quiere reducir el tamaño tumoral y la extensión de la infiltración. ${ }^{39,41,46,47}$ Venuta y su equipo ${ }^{28}$ observaron $90 \%$ de supervivencia en pacientes que recibieron quimioterapia de inducción comparada con $71 \%$ en pacientes con escisión primaria.
Las respuestas a esquemas combinados de quimioterapia son mejores que cuando se utiliza una sola droga. ${ }^{48}$ Carlson y sus colegas describieron en 1990 el primer caso de quimioterapia exitoso en un paciente con carcinoma tímico indiferenciado metastásico utilizando el esquema PVB (cisplatino, vinblastina y bleomicina). ${ }^{49}$ Los protocolos basados en el uso de cisplatino han demostrado ser efectivos en el tratamiento, ${ }^{50}$ aunque hay muy pocos protocolos prospectivos y se han utilizados en adultos. En los años 90 del siglo pasado algunos estudios mostraban que la ifosfamida como monoterapia tenía actividad en $50 \%$ de los casos. ${ }^{33}$ El uso de antraciclinas es controvertido por el efecto tóxico incrementado cuando se utilizan junto con radioterapia mediastinal. Algunos autores han utilizado la combinación de cisplatino, doxorrubicina y metilprednisolona. ${ }^{51}$ El esquema ADOC (cisplatino, doxorrubicina, vincristina y ciclofosfamida) ha obtenido respuestas parciales. ${ }^{44,47,52}$ Los mejores resultados en estudios fase II se obtienen con esquema PAC (cisplatino, doxorrubicina y ciclofosfamida) y ADOC. ${ }^{52}$

El carboplatino con paclitaxel ha mostrado eficacia inclusive como primera línea de tratamiento en tumores irresecables. ${ }^{53-56}$ Iwaga y sus colaboradores establecieron supervivencia global de 22.7 meses y tasa de supervivencia a un año de $62 \%$ con este esquema en primera línea en estadios avanzados no resecables. ${ }^{55}$

Morio y Fukuda describieron respuestas parciales y resecciones completas en pacientes tratados con cisplatino y paclitaxel neoadyuvante..$^{57-59}$ Lucchi y su equipo utilizaron un protocolo con cisplatino, doxorrubicina y etopósido, con cirugía y radioterapia posquirúrgica, con supervivencia a 10 años de 48 y $45 \%$ para estadios Masaoka III y IV. ${ }^{48}$ Yoh y sus colegas utilizaron quimioterapia semanal con cisplatino, vincristina, doxorrubicina y etopósido con supervivencia a 2 años de $58 \% .^{50}$ Recientemente se han estu- 
diado algunas alternativas para los tumores que expresan receptores de somatostatina. ${ }^{59,60}$ Hasta $86 \%$ de los carcinomas expresan c-kit. Algunos estudios aislados evaluaron la respuesta del carcinoma tímico al imatinib sin éxito. ${ }^{61,62}$

La estadificación Masaoka y la resección tumoral completa son los factores de pronóstico más importantes en el carcinoma tímico. ${ }^{63}$ También se considera el subtipo histológico, ${ }^{44,64}$ la resección quirúrgica ${ }^{30}$ y en adultos el estado de Karnofsky. ${ }^{44}$ La supervivencia promedio en adultos es de 2.5 años. ${ }^{3,7,65}$ Con supervivencias a 5 y 10 años de 40 y $33 \%$, respectivamente. ${ }^{66}$ El subtipo poco diferenciado tiene el peor pronóstico entre los demás subtipos histológicos ${ }^{65}$ con un promedio de supervivencia de $20 \pm 18$ meses. ${ }^{21}$ La mayoría de los casos pediátricos son fatales por progresión. ${ }^{3}$ El grupo polaco informó 7 defunciones de 9 pacientes, uno de ellos por toxicidad de la quimioterapia. $^{9}$

Se presentan dos casos de carcinoma tímico diagnosticados y tratados en el Instituto Nacional de Pediatría (México).

\section{CASO CLÍNICO 1}

Adolescente de 15 años con historial de seis meses que inició con vómito, anorexia, fatiga, pérdida de peso $(10 \mathrm{Kg})$, dolor torácico, cianosis peribucal y ungueal. Clínicamente se encontró soplo sistólico; el examen de sangre reveló hipercalcemia de $16.2 \mathrm{mg} / \mathrm{dL}$ que se trató con alendronato. Se sospechó una cardiopatía congénita. Un ecocardiograma mostro disminución de calibre de ramas pulmonares. Una tomografía axial computada de tórax y una angiorresonancia revelaron un tumor mediastinal anterior que desplazaba la silueta cardiaca; en estrecha relación con los grandes vasos infiltraba el pericardio y la pleura; los ganglios se veían aumentados de tamaño (Figura 1). Con una toracoscopia diagnóstica y la toma de biopsia se pudo diagnosticar carcinoma. Se realizó una esternotomía media y se hizo la resección parcial del tumor y pericardiotomía. El examen histológico del tumor confirmó el diagnóstico de carcinoma tímico (Figura 2). Se inició tratamiento con quimioterapia: CDDP $\left(90 \mathrm{mg} / \mathrm{m}^{2}\right)$; doxorrubicina $\left(60 \mathrm{mg} / \mathrm{m}^{2}\right)$; ciclofosfamida $\left(1500 \mathrm{mg} / \mathrm{m}^{2}\right)$ y prednisona (100 mg/día durante 5 días), recibiendo tres ciclos. Después del primer ciclo el paciente cayó en choque séptico y paro cardiorrespiratorio. Se había reducido en $50 \%$ el volumen del tumor visualizado por imagen. Después del tercer ciclo

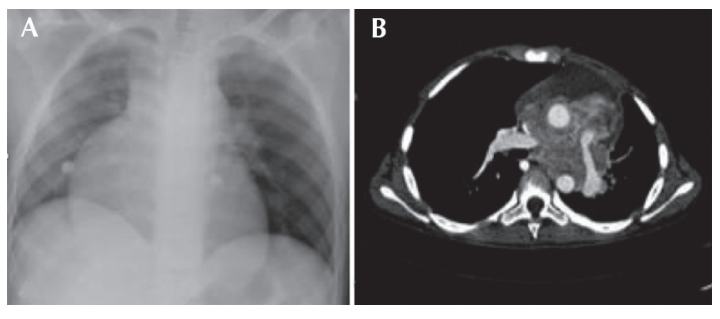

Figura 1. Estudios de imagen que muestran el tumor mediastinal. A) Radiografía de tórax: ensanchamiento mediastinal. B) Resonancia magnética de tórax: tumor mediastinal que desplaza los grandes vasos.

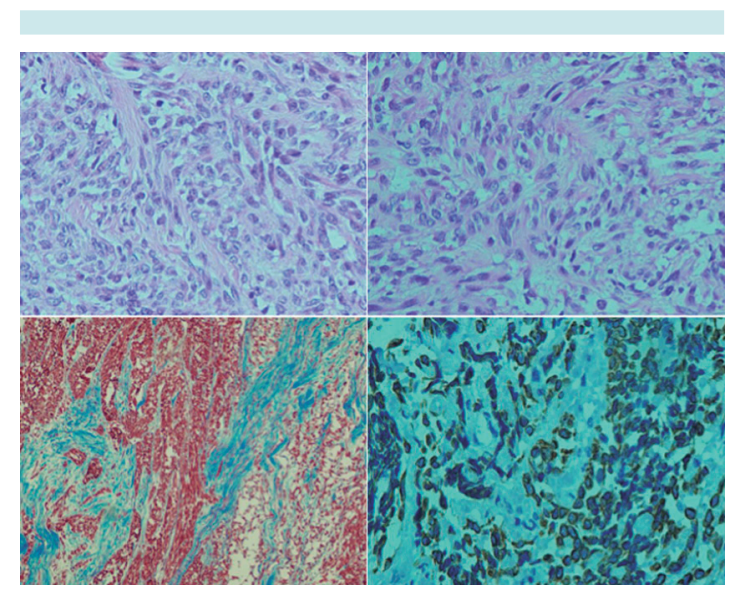

Figura 2. Microfotografías: neoplasia epitelial de células fusiformes con moderado pleomorfismo (H-E). Las células neoplásicas muestran inmunorreactividad positiva para citoqueratina en el citoplasma. 
se produjo bradicardia sinusal, por lo que se suspendió la quimioterapia. Recibió radioterapia con 50.4 Gy. Una tomografía de tórax no mostró evidencia de actividad tumoral. Solamente se vigiló al paciente que falleció 30 meses a causa de una neumonía por Acinetobacter iwoffii. No había evidencia clínica, radiológica ni por patología de actividad tumoral (Figura 1).

\section{CASO CLÍNICO 2}

Niña de 14 años de edad con historia de 3 meses de disnea progresiva y dolor torácico, fiebre y pérdida de peso de $3 \mathrm{~kg}$. Fue tratada como con neumonía sin experimentar mejoría. Una tomografía de tórax mostró un tumor mediastinal anterior con calcificaciones, un derrame pleural y adenomegalias infradiafragmáticas. Se le dio tratamiento con prednisona $(40 \mathrm{mg} / \mathrm{kg} / \mathrm{do})$ debido a los síntomas respiratorios con lo que disminuyó 30\% el tamaño del tumor. Se realizó la resección parcial de $50 \%$ del tumor. Se trataba de un carcinoma linfoepitelial con invasión pleural y pulmonar. Se inició quimioterapia con CDDP (100 mg/m² por 5 días) y 5 -fluorouracilo $\left(100 \mathrm{mg} / \mathrm{m}^{2}\right.$ por 5 días) por 6 ciclos. Posteriormente se realizó radioterapia con 50.4 Gy en el cuello y en el mediastino con lo que hubo una respuesta favorable según mostró una tomografía. Ocho meses después apareció una metástasis pulmonar y hubo que realizar resección. Actualmente la paciente está con enfermedad pulmonar.

\section{ANÁLISIS}

Los carcinomas tímicos en niños son poco frecuentes. Presentamos dos casos diagnosticados en pacientes adolescentes en el Instituto $\mathrm{Na}$ cional de Pediatría en los últimos 10 años. En el primer caso el cuadro clínico correspondió a obstrucción al flujo de salida de los grandes vasos e hipocalcemia, mientras que el segundo correspondió con obstrucción de la vía aérea.
Según la clasificación de Masaoka ambos casos eran avanzados (IVA y IVB, respectivamente) y con resección incompleta inicial, por lo que estaba indicado tratamiento adyuvante. El esquema de quimioterapia utilizado en el caso 1 con CDDP/doxorrubicina/ciclofosfamida ya ha sido demostrado como útil en el tratamiento de carcinoma tímico con residual macroscópico, aunque resultó ser muy tóxico. El uso de radioterapia, indicado en pacientes con residual macroscópico se corrobora en este paciente en el que, a pesar de haber fallecido, no se encontraron datos de actividad tumoral. El esquema utilizado en el caso 2 es un esquema poco utilizado y que no ha demostrado ser efectivo en este paciente que actualmente se encuentra con progresión de la enfermedad.

El carcinoma tímico debe de ser considerado entre los diagnósticos diferenciales de los tumores mediastinales como una causa rara y potencialmente fatal. El diagnóstico temprano, que favorece la resección quirúrgica total, ayudará a disminuir la mortalidad debida a este tumor.

\section{REFERENCIAS}

1. Hasserjian RP, Strobel P, Marx A. Pathology of thymic tumors. Semin Thorac Cardiovasc Surg 2004;17:2-11.

2. de Jong $W K$, Blauwgeers JL, Schaapveld $M$, et al. Thymic epithelial tumours: a population-based study of the incidence, diagnostic procedures and therapy. Eur J Cancer 2008;441:123-130.

3. Yaris N, Nas Y, Cobanoglu U, Yavuz MN. Thymic carcinoma in children. Pediatr Blood Cancer 2006;47:224-227.

4. Smit EF. Thymic malignancies. Ann Oncol 2008;19:309-12.

5. Ramon Cajal S, Suster S. Primary thymic epithelial neoplasms in children. Am J Surg Pathol 1991;15:466-474.

6. Okomura $M$, Schinichiro $M$, Fujii $Y$, et al. Clinical and functional significance of WHO classification on human thymic epithelial neoplasm: A study of 146 consecutive tumors. Am J Surg Pathol 2001;25:103-110.

7. Rios A, Torres J, Galindo PJ, et al. Prognostic factors in thymic epithelial neoplasms. Eur J Cardiothorac Surg 2002;21:307-313.

8. Chen G, Marx A, Wen-Hu C, et al. New WHO histologic classification predicts prognosis of thymic epithelial tumors: $\mathrm{A}$ 
clinicopathologic study of 200 thymoma cases from China. Cancer 2002;95:420-429.

9. Stachowicz-Stencel T, Bien E, Balcerska A, Godzinski J, Synakiewicz A, Madziara W, et al. Thymic carcinoma in children: a report from the Polish Paediatric Rare Tumours Study. Pediatr Blood Cancer 2010;54:916-920.

10. Carretto E, Inserra A, Ferrari A, et al. Epithelial thymic tumours in paediatric age: a report from the TREP Project. Orphanet J Rare Dis 2011;6:28-35.

11. Suster $S$, Moran CA. Thymoma classification: current status and future trends. Am J Clin Pathol 2006;125:542-554.

12. Hishima $T$, Fukayama $M$, Fujisawa $M$, et al. CD5 expression in thymic carcinoma. Am J Pathol 1994;145:268-275.

13. Suster S. Thymic carcinoma: update of current diagnostic criteria and histologic types. Semin Diagn Pathol 2005;22:198-212.

14. Rosai J, Sobin LH. Histological typing of tumours of the thymus. International histological classification of tumours, 2nd edition. New York: Springer, 1999.

15. Schmidt-Wolf IG, Rockstroh JK, Schuller H, et al. Malignant thymoma: Current status of classification and multimodality treatment. Ann Hematol 2003;82:69-76.

16. Liu, H, Hsu W, Chen Y, et al. Primary thymic carcinoma. Ann Thorac Surg 2002;15:1076-81.

17. Niehues $\mathrm{T}, \mathrm{Harms} \mathrm{D}$, Jurgens $\mathrm{H}$, et al. Treatment of pediatric malignant thymoma: Long-term remission in a 14-year-old boy with EBV-associated thymic carcinoma by aggressive, combined modality treatment. Med Pediatr Oncol 1996;26:419-424.

18. Ihan I, Kutluk T, Gogus S, et al. Hypertrophic pulmonary osteoarthropathy in child with carcinoma: An unusual presentation in childhood. Med Pediatr Oncol 1994;23:140-143.

19. Di-Cataldo A, Villari L, Milone $P$, et al. Thymic carcinoma, systemic lupus erytematosus, and hypertrophic pulmonary osteoarthropathy in an 11-year-old boy: A novel association. Pediatr Hematol Oncol 2000;17:701-706.

20. Sung YM, Lee KS, Kim BT, et al. 18F-FDG PET/CT of thymic epithelial tumors: usefulness for distinguishing and staging tumor subgroups. J Nucl Med 2006;47:1628-34.

21. Hartmann CA, Roth C, Mink C, et al. Thymic carcinoma: Report of five cases and review of the literature. J Cancer Res Clin Oncol 1990;116:69-82.

22. Okumura M, Ohta M, Tateyama H, et al. The World Health Organization histologic classification system reflects the oncologic behavior of thymoma: a clinical study of 273 patients. Cancer 2002;94:624-632.

23. Masaoka A, Yamakawa $\mathrm{Y}$, Niwa $\mathrm{H}$, et al. Thymectomy and malignancy. Eur J Cardiothoracic Surg 1994;8:251.

24. Suster S, Moran CA. Thymic carcinoma: Spectrum of differentiation and histologic types. Pathology 1998;30:111122.

25. Muller-Hermelink HK, Marino M, Palestro G, et al. Immunohistological evidences of cortical and medullary differentiation in thymoma. Virchows Arch A Pathol Anat Histopathol 1985;408:143-161.

26. Kondo K, Yasumasa M. Therapy for thymic epithelial tumors: A clinical study of 1,320 patients from Japan. Ann Thorac Surg 2003;76:878-885.

27. Okereke IC, Kesler KA, Freeman RK, et al. Thymic carcinoma: outcomes after surgical resection. Ann Thorac Surg 2012;93:1668-72.

28. Venuta $F$, Rendina $E A$, Longo $F$, et al. Long-term outcome after multimodality treatment for stage III thymic tumors. Ann Thorac Surg 2003;76:1866-1872.

29. Rajan A, Giaccone G. Treatment of advanced thymoma and thymic carcinoma. Curr Treat Options Oncol 2008;9:277287.

30. Yasuda M, Hanagiri T, Oka S, Uramoto H, Takenoyama M, yasumoto K. Results of a surgical resection for patients with thymic carcinoma. Scandinavian Journal of Surgery 2011;100:159-163.

31. Dhall G, Ginsburg HB, Bodenstein L, Fefferman NR, Greco MA, Chang MW, Gardner S. Thymoma in children: report of two cases and review of literature. J Pediatr Hematol Oncol 2004;26:681-685.

32. Liang X, Lovell MA, Capocelli KE, Albano EA, Birch S, Keating AK, Graham DK. Thymoma in children: report of 2 cases and review of the literature. Pediatr Develop Pathol 2010;13:202-208.

33. Giaccone G. Treatment of malignant thymoma. Curr Opin Oncol 2005;17:140-146.

34. Papadopoulos K, Thomas CR. Current chemotherapy options for thymic epithelial neoplasms. Expert Opin Pharmacother 2005;6:1169-1177.

35. Wright, C. D. Extended resections for thymic malignancies. Journal of thoracic oncology: official publication of the International Association for the Study of Lung Cancer 2010;5(10) Suppl 4:S344-7.

36. Ettinger D, Wallace A, Bepler G, Blum M, Chang A, Cheney $\mathrm{R}$, Chirieac $\mathrm{L}$, et al. Thymic Malignancies. Journal of the National Comprehensive Cancer Network. 2010;8:13021315.

37. Rena O, Papalia E, Oliaro A, et al. Does adjuvant radiation therapy improve disease-free survival in completely resected Masaoka stage II thymoma? Eur J Cardiothoracic Surg 2007;31:109-113.

38. Onuki T, Ishikawa S, Yamamoto T, et al. Pathologic radioresponse of preoperatively irradiated invasive thymomas. J Thorac Oncol 2008;3:270-276.

39. Odriozola JM, Muguruza-Trueba I, Muñoz-Molina G, Cabañero-Sánchez A, Saldaña-Garrido D, Lago-Viguera J. Carcinoma tímico. Tratamiento quirúrgico tras quimioterapia neoadyuvante. Neumología y cirugía de tórax 2006;65(4):170-173.

40. Fuller CD, Ramahi E, Aherne N, Eng T, Thomas Jr C. Radiotherapy for thymic neoplasms. Journal of thoracic oncology. 
Official publication of the International Association for the Study of Lung Cancer 2010 5, No. 1004: S327.

41. Wright CD, Choi NC, Wain JC, et al. Induction chemoradiotherapy followed by resection for locally advanced Masaoka stage III and IVA thymic tumors. Ann Thorac Surg 2008;85:385-389.

42. Nonaka T, Tamami $\mathrm{Y}$, Higuchi $\mathrm{K}$, et al. The role of radiotherapy for thymic carcinoma. Jpn J Clin Oncol 2004;34:722-726.

43. Kim ES, Putnam JB, Komaki R, et al. Phase II study of a multidisciplinary approach with induction chemotherapy, followed by surgical resection, radiation therapy, and consolidation chemo-therapy for unresectable malignant thymomas: Final report. Lung Cancer 2004;44:369-379.

44. Ogawa K, Toita T, Uno T, Fuwa N, Kakinohana Y, Kamata M, Koja K, Kinjo T, Adachi G, Murayama S. Treatment and prognosis of thymic carcinoma: a retrospective analysis of 40 cases. Cancer 2002;94(12):3115-9.

45. Eng TY, Thomas Jr C. Radiation therapy in the management of thymic tumors. En Seminars in thoracic and cardiovascular surgery, No 1, WB Saunders. 2005;17:32-40.

46. Yano M, Sasaki H, Yokomyama T, et al. Thymic carcinoma: 30 Cases at a single institution. J Thorac Oncol 2008;3:265-269.

47. Evans T, Lynch T. Role of chemotherapy in the management of advanced thymic tumors. Semin Thorac Cardiovasc Surg 2005;17:41-50.

48. Lucchi M, Ambrogi MC, Duranti L, Basolo F, Fontanini G, Angeletti CA, Mussi A. Advanced stage thymomas and thymic carcinomas: results of multimodality treatment. Ann Thorac Surg 2005;79:1840-1844.

49. Carlson RW, Dorfman RF, Sikic BI. Successful treatment of metastatic thymic carcinoma with cisplatin, vinblastine, bleomycin and etoposide chemotherapy. Cancer 1990;66:2092-4.

50. Yoh, K, Goto, K, Ishii, G, et al. Weekly chemotherapy with cisplatin, vincristine, doxorubicin, and etoposide is an effective treatment for advanced thymic carcinoma. Cancer 2003;98,926-931.

51. Yokoi K, Matsuguma H, Nakahara R, et al. Multidisciplinary treatment for advanced invasive thymoma with cisplatin, doxorubicin, and methylprednisolone. J Thorac Oncol 2007; 2:73-78.

52. Fornasiero A, Daniele O, Ghiotto C, Piazza M, Fiore-Donati L, Calabro F, et al. Chemotherapy for invasive thymoma. A 13-year experience. Cancer 1991;68:30-3.

53. Furugen $\mathrm{M}$, Sekine I, Tsuta K, et al. Combination chemotherapy with carboplatin and paclitaxel for advanced thymic cancer. Jpn J Clin Oncol 2011;41:1013-1016.
54. Maruyama R, Suemitsu R, Okamoto T, Kojo M, Aoki $\mathrm{Y}$, Wataya $\mathrm{H}$, et al. Persistent and aggressive treatment for thymic carcinoma. Results of a single-institute experience with 25 patients. Oncology 2006;70:325-9.

55. Igawa S, Murakami H, Takahashi T, Nakamura Y, Tsuya A, Naito $T$, et al. Efficacy of chemotherapy with carboplatin and paclitaxel for unresectable thymic carcinoma. Lung Cancer 2010;67:194-7.

56. Lemma GL, Lee JW, Aisner SC, Langer CJ, Tester WJ, Johnson $\mathrm{DH}$, et al. Phase II study of carboplatin and paclitaxel in advanced thymoma and thymic carcinoma. J Clin Oncol 2011;29:2060-5.

57. Morio A, Nakahara $K$, Ohse $Y$, et al. Efficacy of induction chemoradiotherapy in thymic cancer: report of a successful case and review of the literature. Int J Clin Oncol 2002;7:201-204.

58. Shintani $\mathrm{Y}$, Ohta M, Hazama K, et al. Thymic carcinoma successfully resected with superior vena cava after chemoradiotherapy. Jpn J Thorac Cardiovasc Surg 2001;49:712-721.

59. Lucchi M, Mussi A, Basolo F, Ambrogi MC, Fontani G, Angeletti CA. The multimodality treatment of thymic carcinoma. European Journal of Cardiothoracic Surgery 2001;19:566569.

60. Kurup A, Loehrer PJ Sr. Thymoma and thymic carcinoma: therapeutic approaches. Clin Lung Cancer 2004;6(1):28-32.

61. Strobel P, Hartmann M, Jakob A, et al. Thymic carcinoma with overexpression of mutated KIT and the response to imatinib. N Engl J Med 2004;350:2625-2626.

62. Pan CC, Chen PC, Chiang H et al. KIT (CD117) is frequently overexpressed in thymic carcinomas but is absent in thymomas. J Pathol 2004;202:375-381.

63. Wright CD, Wain JC, Wong DR, Donhaue DM, Gaissert HA, Grillo HC, Mathisen DJ. Predictors of recurrence in thymic tumors: importance of invasion, World Health Organization histology and size. J Thorac Cardiovasc Surg 2005;130:14131421.

64. Eng YH, Chong JW, Li MS, Hui CC. Post-operative radiotherapy in thymic carcinoma: treatment results and prognosis factors. Int J Radiation Oncology Biol Phys 2002;52(3):801805.

65. Chung DA. Thymic carcinoma: Analysis of nineteen clinicopathological studies. Thorac Cardiov Surg 2000;48:114119.

66. Detterbeck F, Parsons A. Thymic tumors. Ann Thorac Surg 2004;77(5):1860-69. 